\title{
An Adaptive Monitoring Scheme for Automatic Control of Anaesthesia in dynamic surgical environments based on Bispectral Index and Blood Pressure
}

Yu-Ning Yu ${ }^{1}$, Faiyaz Doctor ${ }^{2, *}$, Shou-Zen Fan ${ }^{3}$, Jiann-Shing Shieh ${ }^{1, * *}$

${ }^{1}$ Department of Mechanical Engineering, and Innovation Center for Big Data and Digital

Convergence, Yuan Ze University, Chungli, 320, Taiwan, ROC

${ }^{2}$ School of Computer Science and Electronic Engineering, University of Essex, Wivenhoe

Park, Colchester CO4 3SQ, United Kingdom

${ }^{3}$ Department of Anesthesiology, National Taiwan University Hospital, Taipei, 100, Taiwan

*Corresponding author: Faiyaz Doctor (F. Doctor)

**Co-corresponding author: Jiann-Shing Shieh (J.S. Shieh)

E-Mail: jsshieh@saturn.yzu.edu.tw (J.-S. Shieh), fdocto@ essex.ac.uk (F. Doctor).

Tel.: +886-3-4638800 (ext. 2470); Fax: +886-3-4558013. 
Abstract-During surgical procedures, bispectral index (BIS) is a well-known measure used to determine the patient's depth of anesthesia (DOA). However, BIS readings can be subject to interference from many factors during surgery, and other parameters such as blood pressure (BP) and heart rate (HR) can provide more stable indicators. However, anesthesiologist still consider BIS as a primary measure to determine if the patient is correctly anaesthetized while relaying on the other physiological parameters to monitor and ensure the patient's status is maintained. The automatic control of administering anesthesia using intelligent control systems has been the subject of recent research in order to alleviate the burden on the anesthetist to manually adjust drug dosage in response physiological changes for sustaining DOA. A system proposed for the automatic control of anesthesia based on type-2 Self Organizing Fuzzy Logic Controllers (T2-SOFLCs) has been shown to be effective in the control of DOA under simulated scenarios while contending with uncertainties due to signal noise and dynamic changes in pharmacodynamics (PD) and pharmacokinetic (PK) effects of the drug on the body. This study considers both BIS and BP as part of an adaptive automatic control scheme, which can adjust to the monitoring of either parameter in response to changes in the availability and reliability of BIS signals during surgery. The simulation of different control schemes using BIS data obtained during real surgical procedures to emulate noise and interference factors have been conducted. The use of either or both combined parameters for controlling the delivery Propofol to maintain safe target set points for DOA are evaluated. The results show that combing BIS and BP based 
on the proposed adaptive control scheme can ensure the target set points and the correct amount of drug in the body is maintained even with the intermittent loss of BIS signal that could otherwise disrupt an automated control system.

Index terms- anesthesia; bispectral index; blood pressure; depth of anesthesia; Propofol; type-2 Self Organizing Fuzzy Logic Controllers; pharmacodynamics and pharmacokinetic.

\section{Introduction}

During surgical procedures, anesthesiologist primarily use BIS to determine the patient's DOA, hence it forms a vital element in the effective monitoring and control of anesthesia drug delivery. The anesthesiologist needs to maintain patients in a safe though sufficiently deep state of anesthesia to undergo surgery. It is therefore necessary for them to take into consideration the patients' physiological parameters such as BIS, electrocardiography (ECG), respiration, blood pressure (BP) [1], electromyography (EMG) and electroencephalography (EEG) [2] together with their health condition, physical characteristics, type of surgery performed and the surgical environment to effectively adjust the dosage of drugs. Anesthesiologist still however consider BIS as a primary measure to determine DOA because BIS is considered more accurate for measuring DOA. BIS signals are however prone to interference from various factors: during surgical procedures as compared with the other 
physiological signals (BP, HR and EEG). These can be due to the use of high-frequency electrosurgical devices such as an electric surgical knife that uses high-frequency, alternating polarity and electrical current on biological tissue as a means to cut, coagulate, desiccate, or fulgurate tissue [3]. In situations where there is a loss or corruption of the BIS signal it becomes difficult to continuously adjust and maintain the optimal and safe amount of the drug the patient needs to receive if these adjustments are solely base on BIS alone [4]. Additionally, BIS is only effective as a measure of DoA when the drug affects to cortex [5-7], which means BIS only can measure the signals from the pallium. The most important reason for combining of the physiological signals is that one signal may have some problems such as noise interfere or no effect for some kinds of drugs, during surgery. As a result, this study considers both BIS and BP to adjust dosage of the drug during surgery. If the dosage of the drug (normally either Propofol or Isoflurane) is too high, the patient will feel uncomfortable post-surgical side-effect such as vomiting, dizziness and lethargy. The concentrations of anesthetic drug required will be affected by the patient's physiological characteristics [8] [9] such as age, gender, height and weight. The effects pertaining to the drugs concentration in the body is described by its pharmacodynamics (PD) properties, while the effects related to its concentrations in the bloodstream are describe by pharmacokinetics (PK) properties.

In order to aid the anesthetist in the monitoring and control of DOA, recent research has investigated the design of systems for accurately administering and adjusting the delivery of 
anesthetic in direct response to patients' physiological changes based on intelligently adaptive closed-loop control systems [10-24]. The main limitations of these systems are that BIS is one of the main reference value used to control DOA of patients, which is prone to disruption when the signal is interfered with. There is therefore a need to develop a new adaptive automatic DOA control scheme that can automatically adjust to monitor different parameters in response to changes in the availability and reliability of signals during surgery, to provide undisrupted continuously control of the drug delivery mechanisms.

In this paper $\mathrm{BIS}$ is combined with $\mathrm{BP}$ as the reference values to control DOA of patients. Combing both BIS and BP can compensate for signal interference in BIS, by substituting the BP signal as the reference value to control Propofol infusion. There have been a series of FLCs applications for automatic control of drug infusion as described in [15] [25] [26]. Recently, there have been several studies about using SOFLC for biomedical systems including muscle relaxation [27, 28], DOA [29], and patient analgesia control [30]. The SOFLCs were able to adaptively adjust the multivariate control outputs for the drug concentration and infusion rates, and these studies have been presented in $[31,32]$. In a previous study [33], the authors use both type-1 Self Organizing Fuzzy Logic Controllers (T1-SOFLCs) and type-2 Self Organizing Fuzzy Logic Controllers (T2-SOFLCs) to maintain set points for muscle relaxation and BP. The results showed that T2-SOFLC controller gave a statistically better performance in these control scenarios as compared to the type- 1 based 
controller. The T2-SOFLC has previously also been shown to be effective in the control of DOA under simulated scenarios while contending with uncertainties due to signal noise and dynamic changes in pharmacodynamics and pharmacokinetic effects of the drug on the body [34][35]. We have therefore used the T2-SOFLC as part of the control scheme developed in this study.

We introduce a new adaptive control scheme that can dynamically combine and select BIS and BP as the control reference parameters to control the infusion of Propofol more effectively in the event of loss or interference in BIS signals. Surgery simulations are performed using a T2-SOFLC for anesthesia control based on the comparisons of four control schemes. In the first control scheme, BIS is the only reference value used to affect the target control of the Propofol infusion rate [21] [22] [23], as shown in Fig. 1. This is compared to the second scheme where BP alone is used to affect the control target and the third scheme in which both BIS and BP parameters are used to control the drug infusion. Finally we compare the fourth control scheme in which the reference value is automatically substituted between $\mathrm{BIS}$ and $\mathrm{BP}$ when the BIS signal is lost or interfered is compared. To simulate the expected interference factors affecting the quality of BIS signal in the operating theatre, appropriate levels of noise are added in the simulations. These are derived based on BIS data collected from 72 patients during various types of surgical procedures. For each control scheme the 
study also compares the amount of the drug that is delivered into the body. Results show that the proposed new control scheme is able to maintain the target set points for BIS, BP and the correct amount of drug in the body over the course of surgery being simulated. The rest of the paper is organized as follows: In Section 2, we describe the patient anesthetic model and the derived reference model which we use in our anesthesia control simulations for evaluating the performances of the different control schemes; Section 3 we describe how the T2-SOFLC is used for automatic control of anaesthesia by enabling a physiological characterd-loop control of drug infusion based on BIS and BP parameters; In section 4 we describe the simulation schemes for controlling the infusions rate of Propofol to maintain the DOA of the patient during surgery to determine the best control combination of BIS and BP signals for effective monitoring of the patients' status of anesthesia; Experimental results are presented in section 5 where the different schemes for combining BIS and BP signals are evaluated based on simulated surgical scenarios; finally conclusions are presented in section 6 .

\section{Patient Anesthetic Model}

The anaesthesia model [34], consists of three components: muscle relaxation, unconsciousness and analgesia [36] and is combined with Pharmacokinetics (PK) and Pharmacodynamics (PD) of the three compartments [35]. This PK-PD model is able to measure the drugs interactions with the body to determine not only the DOA but also the 
value of BP and the muscle relaxation [37] [38].

Previous research has shown that BIS can be used to determine the DOA of the patient more precisely [39] [40] and a similar anaesthesia model based on using BIS as a reference parameter for modelling the interaction of anaesthesia with the human body has been shown in [34] [35]. Isoflurane has been widely used in the past for regulating DOA through effecting BP and Muscle relaxation [9] [41-43]. However, this has recently been replaced with Propofol, which can improve patients' recovery time and postoperative effects such as vomiting. In this study, four kinds of control schemes based on the combinations of BIS and BP parameters for controlling delivery Propofol under different surgical operating conditions are evaluated. Previous work in [6-9] [35], has mostly only used BIS for controlling anesthesia delivery. If BIS is interfered by other factors, the infusion rate of Propofol cannot be effectively controlled. Hence these approaches can suffer from control inaccuracies in anaesthesia delivery when the BIS single is interfered and disrupted from surgical equipment and environmental factors. These inaccuracies in the amounts of drug delivered into the body can affect patients' postoperative recovery. The proposed approach uses both BIS and BP to mitigate the effects of BIS signal disruptions and offers different control schemes for being able to adapt to various situations during clinical surgery. 


\subsection{The Propofol Mathematical Model}

Based on previous studies [38] Propofol's pharmacokinetics characteristics can be described by equation (3) in terms of the following three-compartment model:

$$
\begin{gathered}
\dot{x_{1}}=-\left(k_{10}+k_{12}+k_{13}\right) x_{1}+k_{21} x_{2}+k_{31} x_{3}+u \\
\dot{x_{2}}=k_{12} x_{1}-k_{21} x_{2} \\
\dot{x_{3}}=k_{13} x_{1}-k_{31} x_{3}
\end{gathered}
$$

Where $x_{1}, x_{2}$ and $x_{3}$ represent the amount of drug in the central compartment and the peripheral compartments, $u$ infusion rate of Propofol, the constants $\mathrm{k}_{10}$ is the rate of the drug in the central compartment. The other constants $\mathrm{k}_{\mathrm{ij}}$ are calculated by the following equation (4):

$$
\begin{gathered}
k_{12}=\frac{C_{L 2}}{V_{1}}, k_{13}=\frac{C_{L 3}}{V_{1}}, k_{21}=\frac{C_{L 2}}{V_{2}}, k_{31}=\frac{C_{L 3}}{V_{3}} \\
V_{1}=4.27, V_{2}=18.9-0.391(\text { age }-53), V_{3}=238 \\
C_{L 1}=1.89+0.0456(\text { weight }-77)-0.0681(L B M-59)+0.0264(\text { height }-177) \\
C_{L 2}=1.29-0.024(\text { age }-53), C_{L 3}=0.836
\end{gathered}
$$

With lean body mass (LBM) for males calculated as:

$$
L B M=1.1 \text { weight }-128 \frac{\text { weight }^{2}}{\text { height }^{2}}
$$

And LBM for females calculated as: 


$$
L B M=1.07 \text { weight }-148 \frac{\text { weight }^{2}}{\text { height }^{2}}
$$

The Propofol effect-site concentration can be related to the compartment- 1 concentration based on the following equation (10) [52]:

$$
\dot{C}_{e}=k_{e o}\left(C_{p}-C_{e}\right)
$$

Where $C_{p}$ can be obtained by dividing $x_{1}$ with $V_{l}, \mathrm{C}_{\mathrm{e}}$ is the effect-site compartment concentration and $k_{e o}$ is a constants, which is equal $0.456 \mathrm{~min}^{-1}$. Based on the above, the BIS can be calculated by the following equation (11):

$$
B I S=\left(E_{0}-E_{\max } \frac{C_{e}^{\alpha}}{C_{50}^{\alpha}+C_{e}^{\alpha}}\right)
$$

Where $E_{0}=100, E_{\max }=100, C_{50}=7.5 \mathrm{mg} / \mathrm{l}, \alpha=3$.

\subsection{Data Collection from Patients}

In this study, the BIS signals were collected from seventy-two patients, whose ages ranged from twenty to eighty years old, with heights ranging from one hundred and forty to one hundred and eighty centimeters, and weights ranging from thirty to one hundred kilograms. These patients all had diverse illnesses and had to undergo different types of surgical procedures, requiring the administering of general anesthesia. The equipment in the operating room included a physiological monitor (Philips IntelliVue MP60) and a portable computer which was used to displays the patient's physiological signals, specifically: ECG, EEG, BIS, BP and saturated percentage of oxygen $(\mathrm{SpO} 2)$ in real time. The proposed study is 
aimed at single channel BIS signal analysis, based on a sample frequency of $0.2 \mathrm{~Hz}$ for the interpretation of DOA using the BIS Sensor. The research had been approved by an institutional review board and all the patients signed the informed consent.

\section{Automatic Anesthesia Control based on T2-SOFLC}

Fuzzy logic controllers (FLC) can be used to design robust controllers, which can perform well in the face of various sources of real world environmental and instrumentational uncertainties. Moreover, FLCs are able to display robustness relevant to noise and variation of system parameters in complex highly non-linear problem domains such as biomedical control systems. FLCs have been used in several recent applications for automatic control of anaesthesia [44-50] where providing to enable static and adaptive closed-loop control of drug delivery that have been evaluated through simulated surgical scenarios. The T2-SOLFC [48] is a hierarchical adaptive fuzzy controller that is able to generate and modify its rule-base in response to its control performance. Type-2 SOFLCs uses uncertain fuzzy quantifiers constructed from real surgical data to capture patient variability in monitored physiological parameters during anesthetic sedation. Fig.1 shows a diagram of the proposed closed loop anesthesia delivery system that incorporates the type-2 SOFLC for evaluating the four different control schemes.

T2-SOLFC is an adaptive control including fuzzification, inference, control rules, type 
reduction as well as defuzzification, as displayed in Fig. 1. The input values are taken from the patient's anesthetic model outputs minus the set points for the parameters being regulated. These input values are error of BP, the integration error of BP, the error of BIS and the integration error of BIS in order to control DOA of the patient. There is an output control signal in accordance with changing Propofol infusion rate, which is based on the integration of the output value for the purpose to promote real-time adjustment of anesthetic drug. The controller output is transformed back to real values, using an output-scaling element and sent to the patient anaesthetic model. The output of the patient model is compared to the set points for BIS and BP to calculate the error and integration error of the input control signals which are then fed back to the type-2 SOFLC to generate any adjustments to the infusion rate.

\section{Surgical Simulations to Evaluate BIS/BP Control Schemes}

The major idea of this study was to simulate controlling the infusions rate of Propofol for maintaining the DOA of the patients more accuracy during surgery while determining the best choice and combination of key physiological signals for effective monitoring of the patients' anesthetic state. The input signal from the patient anaesthetic model to T2-SOFLC controller is taken at each sampling instant in the form of BIS and BP inputs values for which specific set points need to be maintain for DOA control. The anaesthetic model decides signal values for BIS and BP based on the interaction of Propofol 
infusion rate that is output by the controller. Noise is added to the model signals which are then compared with reference signals for BP and BIS generated from a previous control actuation. The error and integration of error for each signal is calculated to form the closed loop inputs to the controller. Propofol is the output control signal, which is adjusted based on these input values in order to promote real-time revision of drug infusion rates.

The noise added to the BIS signal was based on BIS data collected from seventy-two anesthetized patients during live surgical procedures conducted within a surgical theatre at the National Taiwan University hospital (NTUH). The collected data is based on the average values of BIS over the duration of each patient's surgery. From these values we can then derive the average percentage of BIS $\mu_{B I S}$ and standard deviations $( \pm) \sigma_{B I S}$ for each patient as shown in Table 1. Due to the fact that the noise and the PK and PD results of anesthetic on the patient's body may vary over the duration of the surgery the $( \pm) \sigma_{B I S}$ values for a given patient represent the intra patient variability. Noise related to BP is derived based on a previous study where clinical data was collected from 15 anesthetized patients undergoing ear nose and throat surgical procedures [48] where the average BP value $\mu_{\mathrm{bp}}$ over each of the patients was $90.88( \pm)$ 12.40. The BP data from this previous study sufficiently recorded and considered the environmental conditions effecting the BP readings within a real surgical context. Hence the data was deemed acceptable to be used for approximating noise related to the BP parameter in the proposed system. 
Four different control schemes have been simulated in this study which are: (i) the use of

BIS alone as the reference value for controlling Propofol infusion rates until BIS arrive at a desired set point, (ii) the use of BP alone as the reference value for affecting the control target, (iii) the combined use of both BIS and BP for controlling the Propofol infusion rate and finally (iv) the use of a control selection scheme where BIS is automatically substituted with $\mathrm{BP}$ when the BIS signal gets compromised due to environmental interference.

\section{Simulation Experiments and Results of BIS/BP Control}

\section{Schemes}

The strength of physiological signals like BIS and BP are very weak and susceptible to interference during measurement. In this study, three different strengths of noise at: $0 \%, 10 \%$ and $20 \%$ of standard deviation of the signal derived from the collected patient data were used to simulate real surgical conditions. These values were determined based on previous studies [48]. The range of the BIS is from 100 to 0 . Based on the experience of anesthesiologists, Clinical opinion suggested that BIS should generally be maintained at 40 to 60 during surgery to enable a faster emergence from anesthesia while keeping a lower administered drug dosage [53], which can reduce the risk of adverse postoperative effects. Therefore in our experiments a BIS value of 50 was set as the target set point for the control system to maintain. We have conducted control simulations, which have been evaluated with 30 patients for each of the 
four different control schemes. The simulation flow diagram is shown in Fig. 2. In the following subsections we present the simulation results and analysis for each of the four control schemes where we evaluate each scheme based on the Mean Absolute Error (MAE) to the control set points that needed to be maintained which is calculated using the following equation (12):

$$
\mathrm{MAE}=\frac{1}{n} \sum_{i=1}^{n} \mid \text { value }_{\text {setpoint }}-\text { value }_{\text {error }} \mid
$$

Where $n=$ total number of data, the values of MAE as shown in Table 2 .

\subsubsection{BIS used as the control target}

In this simulation, BIS is used as the only target to control anaesthesia. Fig. 3 shows that BIS is maintained at the desired set point however BP fails to be able to maintain its set point and starts to fall over the course of surgery to potentially dangerous levels. BIS is further subject to different levels of noise affecting the signal, and the results show that the amount of the Propofol comparatively increases with the $\%$ of added noise as shown in Table 2 . This shows that BIS can be interfered by noise and hence cannot effectively be used on its own to control Propofol delivery.

\subsubsection{BP used as the control target}

According to the experience of anesthetists, a patient's BP will diminish from 15 to 20 percent of baseline, which is measured during the induction phase. The result shows that if $\mathrm{BP}$ is required to be maintained at 15 to 20 percent less of baseline, BIS tends to reduce to a 
value of 60, as shown in Fig. 4. Fig. 4 also shows that while BP is maintained steadily at the desired set point, the value of BIS is unable to reach the desired set point. Both the single target control schemes mentioned here and in section 5.1.1 for controlling BIS and BP in isolation fail at effectively maintaining both these parameters within acceptable ranges for safe DOA. Furthermore as both BIS and BP are influenced by Propofol, it is impossible for both parameters to be maintained at their desired set points at the same time as shown in Fig. 3 and Fig. 4 [35] [38].

\subsubsection{BP replaces BIS as the control target when BIS reaches 50}

Based on expert opinion the BIS's values need to be maintained at 40 to 60 . Until a BIS value of around 50 has been achieved surgical practice is to monitor the patients BP followed by, HR or other parameters. BIS and BP should ideally be maintained at their set points together however in practice given this is difficult to achieve then it is acceptable for a tolerable range to be maintained for both these parameters during surgery. The suggested range that BIS should be maintained is maintained at 40 to 60 , whereas $\mathrm{BP}$ should to be maintained at 15 to 20 percent less of baseline based on clinical expert judgment. From the simulations of the previous two control schemes shown in Figs. 5 and 6, we see that if BIS is the only target; BP will be reduced too much becoming dangerously low for the patient. In the case when BP is controlled on its own the patient is unable to achieve suitable DOA. Hence we propose a control scheme in which both BIS and BP are combined together as the 
reference values for controlling the target infusion rate. In this scheme the initial control requirement is to achieve a safe BIS of 50 until which point BP can be used as the control target for drug delivery. The performance of this control scheme however can be affected due to the loss of BIS signals from interferences caused by the use of electrosurgical devices and other disrupting effects during surgery. In such cases Propofol would be inaccurately maintained based on the amount of the drug in the previous control timestamp prior to the BIS signal being disrupted. This situation is shown in Fig. 5.

\subsubsection{BP alternates with BIS as the control target when BIS signal is lost due to interference}

During the surgical procedures, the endotherm knife is commonly used by surgeons to perform incisions on the body as it can reduce the amount of bleeding which occurs. Due to this being a high-frequency tool it can interfere with monitored physiological signals from the patient specifically BIS. When this occurs the streaming BIS value will output a value of -1 . In this control scheme, BIS is substituted with BP for controlling the target infusion rate when it is equal to -1 . Fig. 6 shows that at several points during the simulation the BIS signal is equal to -1 simulating the interference from either noise or high-frequency electrosurgical equipment. In these situations BP is used to control Propofol. When the value of BP is lower than 80 , the infusion rate of Propofol decreases in order to maintain the target for BP. After the BIS signal recovers, the infusion rate of Propofol is increased in order to then maintain 
the target for the BIS signal. Table 2 shows the situations where noise has been added, and the corresponding control behavior. Comparing this control scheme with the third control scheme described in section 5.1.3 the infusion rate of Propofol in that control scheme is shown to be more stable as can be seen in the comparison between Fig. 5 and Fig. 6.

In terms of the evaluations performed on all four of the control schemes the MAE's values of the BIS are smallest in the first control scheme However, the MAE's values of BP in the first scheme is the largest of all the schemes compared. Regarding the second scheme, the MAE's values of BIS is the largest compared to all the other control schemes; however, the MAE's values of BP is able to achieve a good performance. Compared to the first and second control schemes, in the third scheme where we take both BIS and BP in consideration to control Propofol, the MAE's values for BIS and BP are shown to have better results. Comparing with the fourth method, although the MAE's values of BIS in the third control scheme is higher, the MAE's values of BP are much lower and the infusion rate of Propofol in third scheme is also more stable. Hence the third control scheme shows a better performance for managing of Propofol infusion rates compared to the other three schemes.

From our analysis of these four control schemes, the results show that by using BIS alone to control Propofol infusion the amount of drug infused is reduced compared with the control schemes in which both BIS and BP are combined. However when using BIS as the only control target, the system is unable to achieve reliable continuous control of Propofol 
when BIS signal is lost due to interference from environmental conditions. In these circumstances Propofol has to be maintain based on the amount of the drug at the previous timestamp, which compromises the accuracy of drug delivery providing incorrect dosages to changes in the patients' physiological state. The control scheme based on controlling Propofol infusion using BP alone as the control target uses the least amount of drug among the four control schemes. However in this control scheme the value of BIS is unable to reach the desired set point tending only to reduce to 60 . When performing surgical procedures the BIS value should be maintained at between 40 and 60 [4] [6] and in some surgical scenarios the DOA needs to be more deeply induced at values below 60. For these cases the control scheme based on BP being used as the control target is unable to assure the patient is induced to an appropriate DOA.

With regard to the two control schemes that combine BIS and BP for controlling the target infusion of Propofol, both show an ability to control the infusion of Propofol while there are other factors causing the interference of the BIS signal. The first of these two schemes follows the anesthetists monitoring process, which is based on other physiological signals such as BP and HR being used to monitor DOA until the desired BIS set point is reached. This method can let both BIS and BP be maintained within a desired range, which is more suitable to the patients' postoperative recovery. However the scheme can still suffer from the intermittent disruption of BIS signals due to the environmental interferences. In the 
second of these two schemes BP is automatically substituted for the BIS reference variable whenever the BIS signal is lost due to environmental interference. This method enables Propofol delivery to be continuously maintained using BP which can solve the situation in which is Propofol is inaccurately maintained based on the amount of the drug in previous timestamp prior to loss of the BIS signal.

In order to statistically compare differences in performance between each method, Table 3 shows the mean MAE \pm SD values of each control scheme for 30 patients in Table 2 . Here the MAE \pm SD values were calculated by using ANOVA in Matlab to do the statistical analysis of the performance variation of each scheme in comparison to the third control scheme which had shown best performance. Comparing the performances of BIS and BP of the first control scheme with that of the third, there were no significant differences shown between these two methods. This is due to the fact that both control schemes are generally the same except when BP replaces BIS as the control target when BIS reaches 50 in the third control scheme. However, there are still minor differences between these two schemes. For example, the BIS's value under $0 \%$ noise in the first scheme is lower than the third scheme. This is because there are several points in time when infusion is controlled by BP in the third scheme. Moreover, the BP's value under $0 \%$ noise in the first scheme is larger than the third method because of the same reason where at several points in time the control of infusion is based on BP in the third scheme. In terms of comparison between the second and third 
control schemes, there are significant differences between both BIS and BP in these two methods. In the second control scheme, BP is the only control target, therefore, the BP's values in this control scheme are smaller than the third scheme. In addition, the BIS's values in the second control scheme is higher than the third scheme. The performance comparison between the fourth and the third control schemes show significant differences in the values for BP when adding 20\% noise. However as there are only a few points during the simulation when the BIS signal is equal to -1, the values for BIS do not show much of a change in Table 3. A possible reason for significant differences in values BP occurring only under $20 \%$ noise is that most signals may probably between 50 to -1 after adding $20 \%$ noise. Nonetheless, this study shows that generally better performance results are achieved when controlling the infusion rates of Propofol when adding noise that is derived from collected patient data based on real surgical conditions.

\section{Conclusions}

In previous work [5-7], most approaches have used BIS to control anesthesia drug delivery during surgery, given its importance as a clinical index for determining DOA. However in real surgical situations environmental interferences can affect the reliability of the BIS signal. There are many factors which cause interference to the BIS signal such as noise in the sensor readings or interference due to the use of electrosurgical equipment. As a 
result the infusion of Propofol cannot be accurately controlled. In this study, an automatic control scheme based on T2-SOFLCs combining both BIS and BP parameters to maintain the DOA of the patient during surgery has been proposed. Simulations of different control schemes where noise was added based on real surgical data was used to evaluate either or both these combined parameters for controlling the delivery of Propofol to maintain safe target set points for DOA. The results showed that combing BIS and BP can ensure the target set points for BIS and BP can both be maintained at a safe and acceptable range (between 40 to 60 for BIS and 15 to 20 percent less of the baseline for BP) based on the correct delivery of drug even with the intermittent loss of BIS signal. In statistical analysis conducted to compare performance differences between each of the four controls scheme evaluated, neither the first and the third control schemes nor the fourth and the third control schemes showed significant differences, though there were some minor differences seem. However in comparing between the second and the third control schemes there was found to be significant performance differences. However, the third control scheme still showed the best way to control the infusion rate for consider both BIS and BP. In our future work we will investigate combining this adaptive control approach with a model free methodology based on Genetic Algorithms (GA) / Genetic Programming (GP) approaches for constructing a drug interaction model directly derived from patient data to provide a more data sensitive drug modeling and delivery system. 


\section{Compliance with Ethical Standard}

Funding: This study was funded by National Chung-Shan Institute of Science \& Technology in Taiwan (Grant Numbers: CSIST-095-V201 and CSIST-095-V202). Conflict of Interest: Yu-Ning Yu, Faiyaz Doctor, Shou-Zen Fan, Jiann-Shing Shieh each declare that they has no conflict of interest. Ethical approval: All procedures performed in studies involving human participants were in accordance with the ethical standards of the institutional and/or national research committee and with the 1964 Helsinki declaration and its later amendments or comparable ethical standards. Informed consent: Informed consent was obtained from all individual participants included in the study.

\section{References}

[1] W. D. N. Kee, A. Lee, K. S. Khaw, F. F. Ng, M. K. Karmakar, and T. Gin, "A randomized double-blinded comparison of phenylephrine and ephedrine infusion combinations to maintain blood pressure during spinal anesthesia for cesarean delivery: the effects on fetal acid-base status and hemodynamic control," Anesthesia \& Analgesia, vol. 107, pp. 1295-1302, 2008.

[2] C. S. Nunes, T. Mendonca, S. Bras, D. A. Ferreira, and P. Amorim, "Modeling anesthetic drugs' pharmacodynamic interaction on the bispectral index of the EEG: the influence of heart rate," in Engineering in Medicine and Biology Society, 2007. EMBS 2007. 29th Annual International 
Conference of the IEEE, 2007, pp. 6479-6482.

[3] G.-W. Ji, Y.-Z. Wu, X. Wang, H.-X. Pan, P. Li, et al., "Experimental and clinical study of influence of high-frequency electric surgical knives on healing of abdominal incision," in World Journal of Gastroenterology, vol. 12, pp. 4082-4085, 2006.

[4] M. M. da Silva, T. Mendonça, and T. Wigren, "Online nonlinear identification of the effect of drugs in anaesthesia using a minimal parameterization and BIS measurements," in American Control Conference (ACC), 2010, 2010, pp. 4379-4384.

[5] M. T. Alkire, A. G. Hudetz and G. Tononi, "Consciousness and anesthesia," Science, 322: 876-880, 2008.

[6] P. L. Purdon, E. T. Pierce, G. Bonmassar, J. Walsh, P. G. Harrell, J. Kwo, D. Deschler, M. Barlow, R. C. Merhar, C. Lamus, C. M. Mullaly, M. Sullivan, S. Maginnis, D. Skoniecki, H. A. Higgins and E. N. Brown, "Simultaneous electroencephalography and functional magnetic resonance imaging of general anesthesia," Ann. N. Y. Acad. Sci., 1157: 61-70, 2009.

[7] L. J. Velly, M. F. Rey, N. J. Bruder, F. A. Gouvitsos, T. Witjas, J. M. Regis, J. C. Peragut and F. M. Gouin, "Differential dynamic of action on cortical and subcortical structures of anesthetic agents during induction of anesthesia," Anesthesiology, 107: 202-212, 2007.

[8] B. Musizza, S. Ribaric, Monitoring the depth of anaesthesia, Sensors 10 (2010)1089610935. 
[9] M. Mahfouf, A. J. Asbury, and D. A. Linkens, "Unconstrained and constrained generalised predictive control of depth of anaesthesia during surgery," Control Engineering Practice, vol. 11, no. 12 , pp. 1501-1515, 2003.

[10] J. Y. Lan, M. F. Abbod, R. G. Yeh, S. Z. Fan, andJ. S. Shieh, "Intelligent modeling and control in anesthesia," Journal of Medical and Biological Engineering, vol. 32, no. 5, pp. $293-308,2012$.

[11] J. Shieh, M. Abbod, C. Hsu, S. Huang, Y. Han, and S. Fan,"Monitoring and control of anesthesia using multivariable selforganizing fuzzy logic structure," in Fuzzy Systems in Bioinformatics and Computational Biology, pp. 273-295, Springer, Berlin, Germany, 2009.

[12] Y.-C. Chou, M. F. Abbod, J.-S. Shieh, and C.-Y. Hsu, "Multivariable fuzzy logic/self-organizing for anesthesia control," Journal of Medical and Biological Engineering, vol. 30,no. 5, pp. 297-306, 2010.

[13] J.-S. Shieh, S.-Z. Fan, L.-W. Chang, and C.-C. Liu, "Hierarchical rule-based monitoring and fuzzy logic control for neuromuscular block," Journal of Clinical Monitoring and Computing, vol. 16, no. 8, pp. 583-592, 2000.

[14] M. E. Karar and M. A. El-Brawany, “Automated cardiac drug infusion system using adaptive fuzzy neural networks controller," Biomedical Engineering and Computational Biology, vol. 3, pp. 1-11, 2011. 
[15] M.L. Kumar, R. Harikumar, A.K. Vasan, V. Sudhaman, Fuzzy controller for auto-matic drug infusion in cardiac patients, in: Proceedings of the InternationalMultiConference of Engineers and Computer Scientists (IMECS 2009), Citeseer,2009.

[16] J. Agarwal, G. D. Puri, and P. J. Mathew, "Comparison of closed loop vs. manual administration of propofol using the Bispectral index in cardiac surgery," Acta Anaesthesiologica Scandinavica, vol. 53, no. 3, pp. 390-397, 2009.

[17] S. Locher, K. S. Stadler, T. Boehlen et al., "A new closed-loop control system for isoflurane using bispectral index outperforms manual control," Anesthesiology, vol. 101, no. 3, pp. 591-602, 2004.

[18] M. M. R. F. Struys, T. de Smet, S. Greenwald, A. R. Absalom, S. Bing'e, and E. P. Mortier, "Performance evaluation of two published closed-loop control systems using bispectral index monitoring: a simulation study," Anesthesiology, vol. 100, no. 3, pp. 640$647,2004$.

[19] V. Esmaeili, A. Assareh, M. B. Shamsollahi, M. H. Moradi, and N. M. Arefian, "Estimating the depth of anesthesia using fuzzy soft computation applied to EEG features," Intelligent Data Analysis, vol. 12, no. 4, pp. 393-407, 2008.

[20] J.-S. Shieh, L.-W. Chang, S.-Z. Fan, C.-C. Liu, and H.-P. Huang, "Automatic control of anaesthesia using hierarchical structure," Yixué gongchéng. Applications, basis, communications, vol. 10, pp. 195-202, 1998. 
[21] N. Liu, T. Chazot, A. Genty, A. Landais, A. Restoux, K. McGee, et al., "Titration of propofol for anesthetic induction and maintenance guided by the bispectral index: closed-loop versus manual control: a prospective, randomized, multicenter study," Anesthesiology, vol. 104, pp. 686-695, 2006.

[22] A. Morley, J. Derrick, P. Mainland, B. Lee, and T. Short, "Closed loop control of anaesthesia: an assessment of the bispectral index as the target of control," Anaesthesia, vol. 55, pp. 953-959, 2000.

[23] A. Absalom and G. Kenny, "Closed-loop control of propofol anaesthesia using bispectral index ${ }^{\mathrm{TM}}$ : performance assessment in patients receiving computer-controlled propofol and manually controlled remifentanil infusions for minor surgery†,," British Journal of Anaesthesia, vol. 90, pp. 737-741, 2003.

[24] S. Locher, K. S. Stadler, T. Boehlen, T. Bouillon, D. Leibundgut, P. M. Schumacher, et al., "A new closed-loop control system for isoflurane using bispectral index outperforms manual control," Anesthesiology, vol. 101, pp. 591-602, 2004.

[25] D.S. Diwase, R.W. Jasutkar, Expert controller for estimating dose of isoflurane,Int. J. Adv. Eng. Sci. Technol. 9 (2011) 218-221.

[26] C. Jiming, C. Kejie, S. Youxian, X. Yang, Continuous drug infusion for diabetestherapy: a closed-loop control system design, EURASIP J. Wirel. Commun. Netw.2008 (2008).

[27] D. Mason, J. Ross, N. Edwards, D. Linkens, C. Reilly, Self-learning fuzzy control of 
atracurium-induced neuromuscular block during surgery, Med. Biol. Eng. Comput. 35 (1997) 498-503.

[28] J. Ross, D. Mason, D. Linkens, N. Edwards, Self-learning fuzzy logic control of neuromuscular block, Br. J. Anaesth. 78 (1997) 412-415.

[29] J. Shieh, D.A. Linkens, A. Asbury, A hierarchical system of on-line advisory for monitoring and controlling the depth of anaesthesia using self-organizing fuzzy logic, Eng. Appl. Artif. Intell. 18 (2005) 307-316.

[30] J.-S. Shieh, L.-W. Chang, T.-C. Yang, C.-C. Liu, An enhanced patient controlled analgesia (EPCA) for the extracorporeal shock wave lithotripsy (ESWL), Biomed. Eng.: Appl. Basis Commun. 19 (2007) 7-17.

[31] J.-S. Shieh, M.F. Abbod, E.D. Krishna, Y.-C. Chou, S.-Z. Fan, The simulation of con-trolling of anesthesia using a novel multivariable fuzzy logic and self-organizing fuzzy logic controller, in: M. Hertzog, Z. Kuhn (Eds.), General Anesthesia Research Developments, Nova Science Publishers Inc., 2009.

[32] J. Shieh, M. Abbod, C. Hsu, S. Huang, Y. Han, S. Fan, Monitoring and control of anesthesia using multivariable self-organizing fuzzy logic structure, in: Fuzzy Systems in Bioinformatics and Computational Biology, Springer, 2009,pp. 273-295.

[33] Y.-X. Liu, F. Doctor, S.-Z. Fan, and J.-S. Shieh, "Performance Analysis of Extracted Rule-Based Multivariable Type-2 Self-Organizing Fuzzy Logic Controller Applied to 
Anesthesia," BioMed research international, vol. 2014, pp. 1-19, 2014.

[34] R. Upton and D. Mould, "Basic Concepts in Population Modeling, Simulation, and Model-Based Drug Development: Part 3-Introduction to Pharmacodynamic Modeling Methods," CPT: pharmacometrics \& systems pharmacology, vol. 3, pp. 1-16, 2014.

[35] P. L. Gambús and I. F. Trocóniz, "Pharmacokinetic-pharmacodynamic modelling in anaesthesia," British journal of clinical pharmacology, vol. 79, pp. 72-84, 2015.

[36] C.-T. Chuang, S.-Z. Fan, and J.-S. SHIEH, "Muscle relaxation controlled by automated administration of cisatracurium," Biomedical Engineering: Applications, Basis and Communications, vol. 18, pp. 284-295, 2006.

[37] J. W. Huang, Y.-Y. Lu, A. Nayak, and R. J. Roy, "Depth of anesthesia estimation and control [using auditory evoked potentials]," Biomedical Engineering, IEEE Transactions on, vol. 46, pp. $71-81,1999$.

[38] D. Ingole and M. Kvasnica, "FPGA Implementation of Explicit Model Predictive Control for Closed Loop Control of Depth of Anesthesia ," 2015.

[39] A. E. Ibrahim, J. K. Taraday, and E. D. Kharasch, "Bispectral index monitoring during sedation with sevoflurane, midazolam, and propofol," Anesthesiology, vol. 95, pp. 1151-1159, 2001.

[40] P. S. Glass, M. Bloom, L. Kearse, C. Rosow, P. Sebel, and P. Manberg, "Bispectral analysis measures sedation and memory effects of propofol, midazolam, isoflurane, and alfentanil in healthy volunteers," Anesthesiology, vol. 86, pp. 836-847, 1997. 
[41] M. El-Bardini and A. M. El-Nagar, "Direct adaptive interval type-2 fuzzy logic controller for the multivariable anaesthesia system," Ain Shams Engineering Journal, vol. 2, no. 3-4, pp. 149-160, 2011.

[42] J. Shieh, M. Abbod, C. Hsu, S. Huang, Y. Han, and S. Fan, "Monitoring and control of anesthesia using multivariable selforganizing fuzzy logic structure," in Fuzzy Systems in Bioinformatics and Computational Biology, pp. 273-295, Springer, Berlin, Germany, 2009.

[43] Y.-X. Liu, F. Doctor, J.-S. Shieh, S.-Z. Fan, and K.-K. Jen "Multivariable type-2 self-organizing fuzzy logic controllers for regulating anesthesia with rule base extraction," in Proceedings of the Conference on Technologies and Applications of Artificial Intelligence, Taipei, Taiwan, 2013.

[44] H. Araujo, B. Xiao, C. Liu, Y. Zhao, and H. Lam, "Design of Type-1 and Interval Type-2 Fuzzy PID Control for Anesthesia Using Genetic Algorithms," Journal of Intelligent Learning Systems and Applications, vol. 6, p. 70, 2014.

[45] S. Bras, L. Ribeiro, D. Ferreira, L. H. M. Antunes, and C. S. Nunes, "Controlling the hypnotic drug (propofol) to maintain a stable depth of anesthesia, in dogs," in Medical Measurements and Applications (MeMeA), 2014 IEEE International Symposium on, 2014, pp. 1-5.

[46] D. Ingole and M. Kvasnica, "FPGA Implementation of Explicit Model Predictive Control for Closed Loop Control of Depth of Anesthesia ," 2015. 
[47] J. A. Méndez, A. Marrero, J. A. Reboso, and A. León, "Adaptive fuzzy predictive controller for anesthesia delivery," Control Engineering Practice, vol. 46, pp. 1-9, 2016.

[48] F. Doctor, C.-H. Syue, Y.-X. Liu, J.-S. Shieh, and R. Iqbal, "Type-2 fuzzy sets applied to multivariable self-organizing fuzzy logic controllers for regulating anesthesia," Applied Soft Computing, vol. 38, pp. 872-889, 2016.

[49] M. Struys, T. De Smet, L. Versichelen, S. Van de Velde, R. Van den Broecke, and E. P. Mortier, "Comparison of closed-loop controlled administration of propofol using Bispectral Index as the controlled variable versus" standard practice" controlled administration," Anesthesiology, vol. 95, pp. 6-17, 2001.

[50] J.-S. Shieh, S.-Z. Fan, L.-W. Chang, and C.-C. Liu, "Hierarchical rule-based monitoring and fuzzy logic control for neuromuscular block," Journal of clinical monitoring and computing, vol. 16, pp. 583-592, 2000.

[51] C. M. Ionescu, R. De Keyser, B. C. Torrico, T. De Smet, M. M. Struys, and J. E. Normey-Rico, "Robust predictive control strategy applied for propofol dosing using BIS as a controlled variable during anesthesia," Biomedical Engineering, IEEE Transactions on, vol. 55, pp. 2161-2170, 2008.

[52] L. B. Sheiner, D. R. Stanski, S. Vozeh, R. D. Miller, and J. Ham, "Simultaneous modeling of pharmacokinetics and pharmacodynamics: application to d-tubocurarine," Clinical pharmacology and therapeutics, vol. 25, pp. 358-371, 1979. 
[53] M. Jeanne, C. Clément, J. De Jonckheere, R. Logier, and B. Tavernier, "Variations of the analgesia nociception index during general anaesthesia for laparoscopic abdominal surgery," Journal of clinical monitoring and computing, vol. 26, pp. 289-294, 2012. 


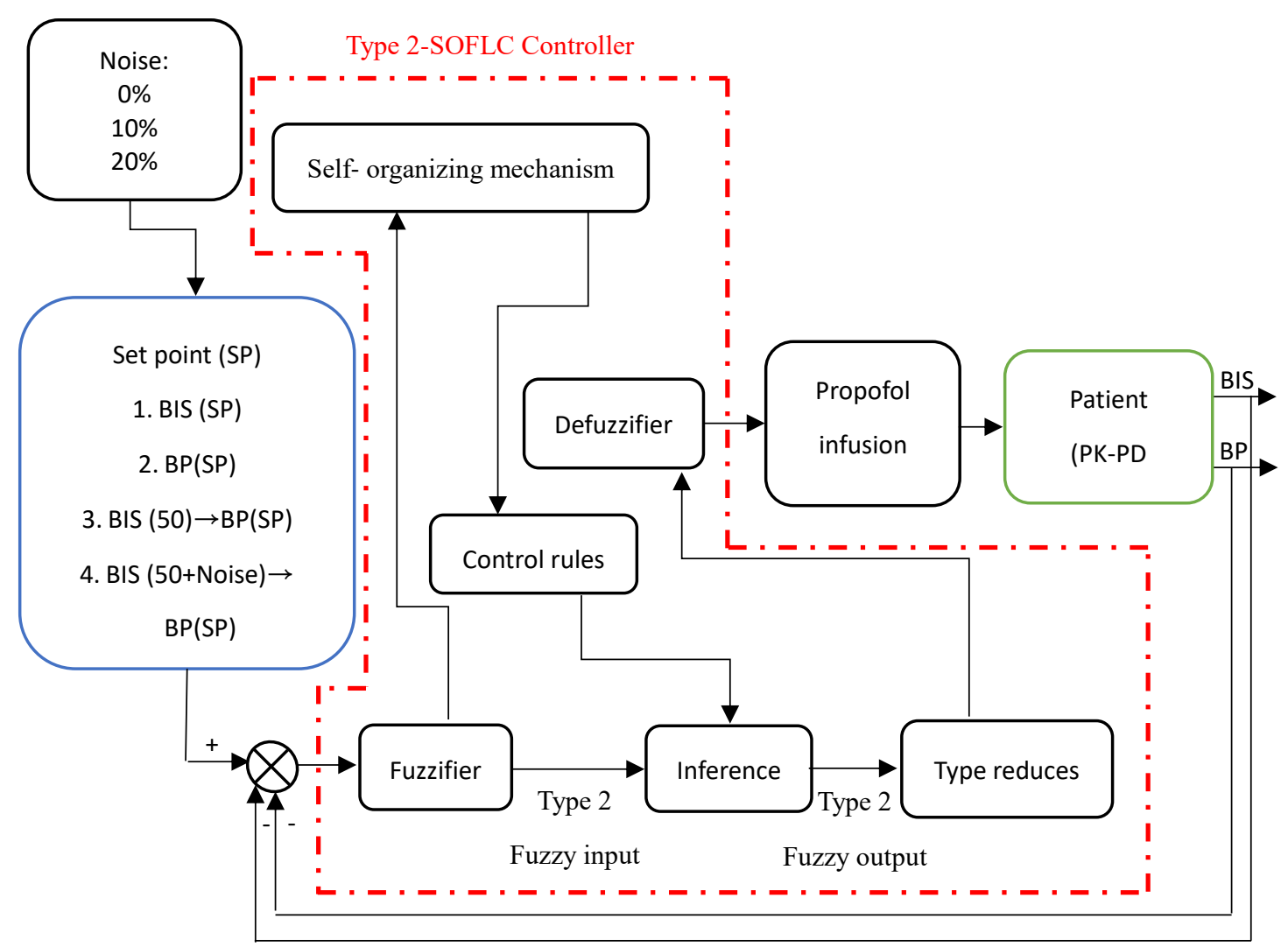

Fig. 1. The structure of the simulation system where 1. BIS (SP): BIS is used as a control target; 2. BP (SP): BP is used as a control target; 3 . BIS (SP) $\rightarrow$ BP(SP): BIS is used as the control target, but replaced by BP when BIS reaches 50; 4. BIS(Noise) $\rightarrow$ BP(SP): BP is used as the control target when BIS signal is lost due to interference from conditions during surgical procedures 


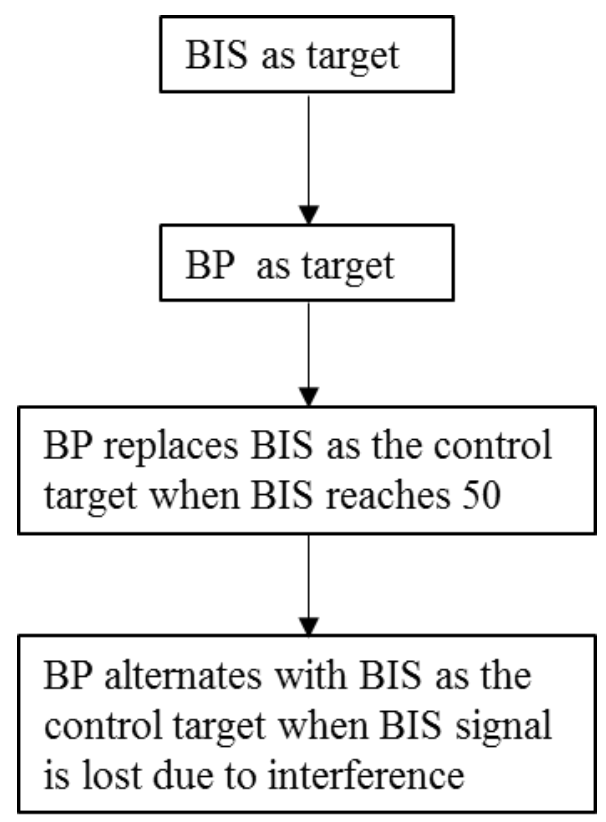

Fig. 2 The simulation flow diagram
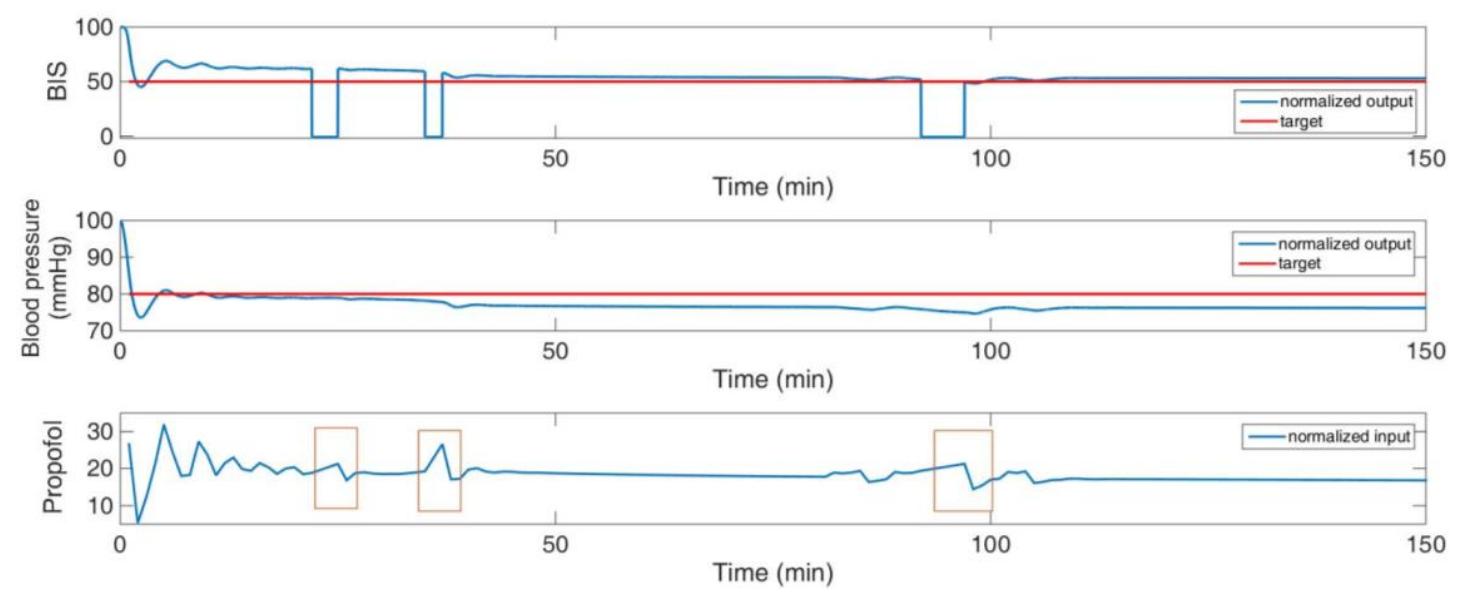

Fig. 3. The simulation results where BIS is used as the control target, under $0 \%$ noise
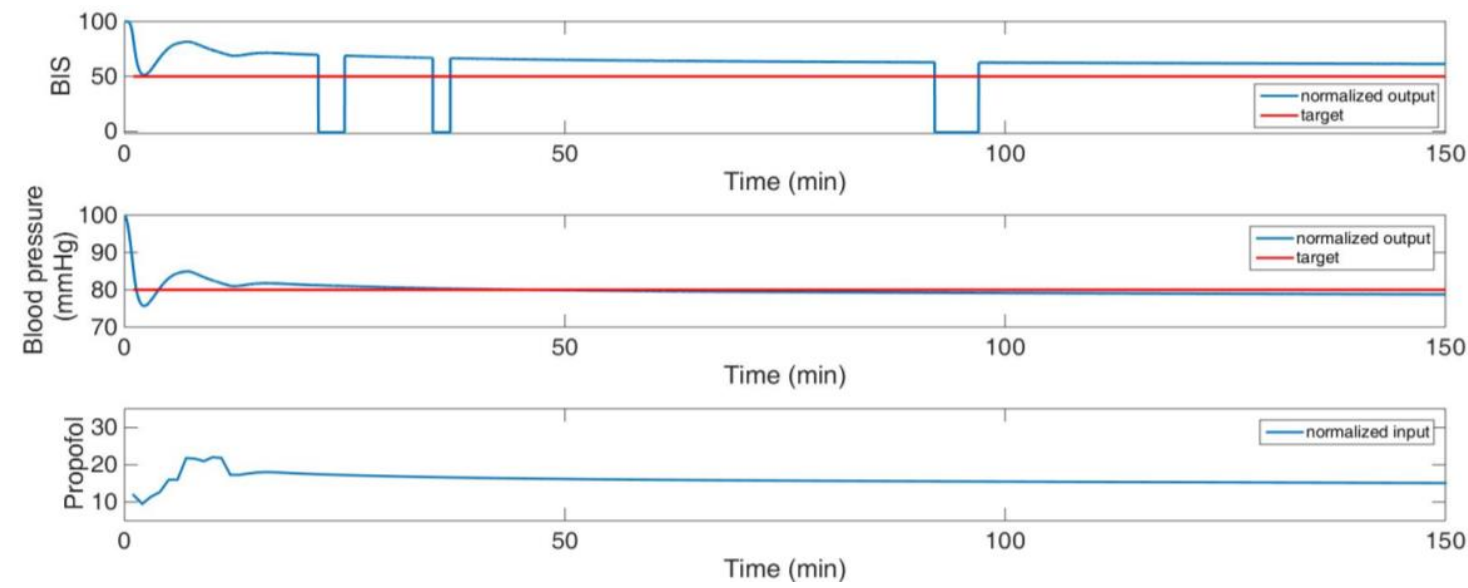

Fig. 4. The simulation result of $\mathrm{BP}$ is used as the control target, under $0 \%$ noise 

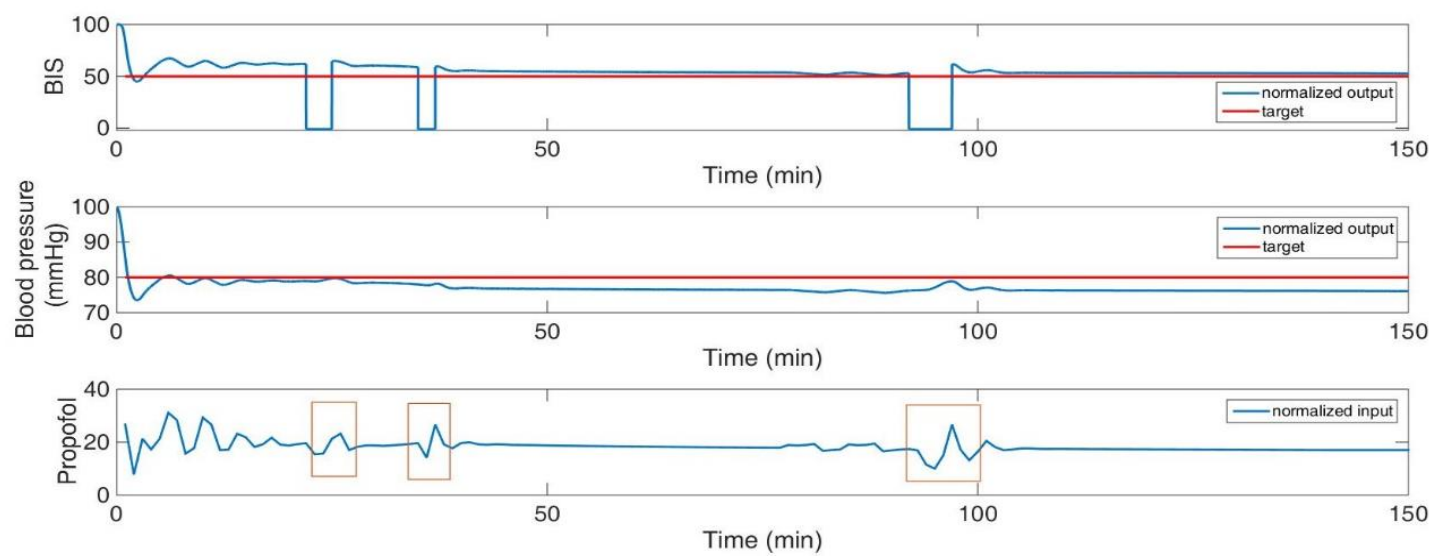

Fig. 5. The simulation results where BP replaces BIS as the control target when BIS reaches 50, under $0 \%$ noise
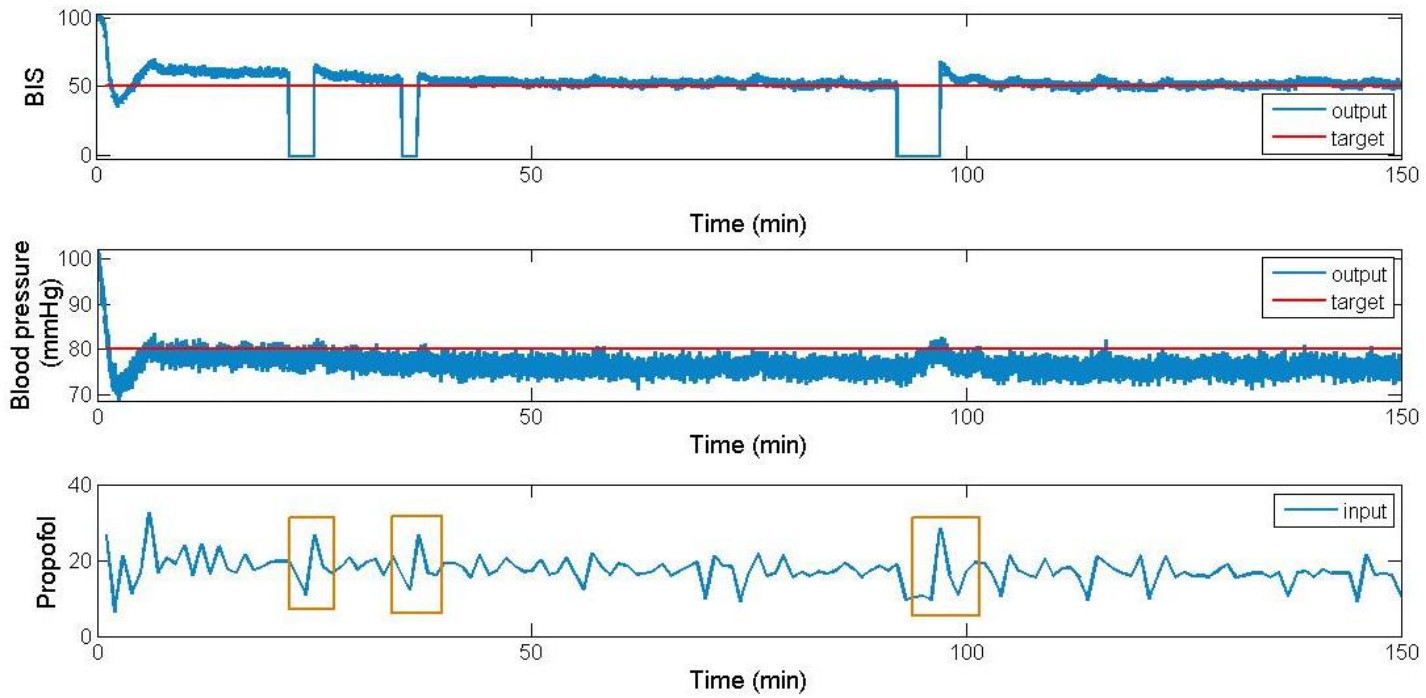

Fig. 6. The simulation result where BP alternates with BIS as the control target when BIS signal is lost due to interference, under $10 \%$ noise 
Table 1. Clinical data from 72 anesthetized patients while undergoing a specific nasal surgical procedures and calculated values for BIS for each patient $(\mu=49.34 \pm 14.19)$

\begin{tabular}{|c|c||c|c||c|c||}
\hline Patient & BIS $\left(\mu_{B I S}, \sigma_{B I S}\right)$ & Patient & BIS $\left(\mu_{B I S}, \sigma_{B I S}\right)$ & Patient & BIS $\left(\mu_{B I S}, \sigma_{B I S}\right)$ \\
\hline 1 & $36.58 \pm 15.28$ & 25 & $30.63 \pm 12.01$ & 49 & $44.47 \pm 16.31$ \\
2 & $41.81 \pm 10.01$ & 26 & $27.34 \pm 14.89$ & 50 & $47.70 \pm 13.45$ \\
3 & $51.93 \pm 11.19$ & 27 & $48.09 \pm 13.50$ & 51 & $49.35 \pm 14.62$ \\
4 & $40.74 \pm 17.23$ & 28 & $62.17 \pm 8.77$ & 52 & $49.49 \pm 14.23$ \\
5 & $38.37 \pm 19.30$ & 29 & $58.30 \pm 22.56$ & 53 & $63.77 \pm 16.01$ \\
6 & $41.59 \pm 14.15$ & 30 & $66.10 \pm 18.08$ & 54 & $37.62 \pm 13.30$ \\
7 & $44.42 \pm 19.17$ & 31 & $52.48 \pm 15.69$ & 55 & $40.33 \pm 13.08$ \\
8 & $44.46 \pm 12.24$ & 32 & $45.99 \pm 14.04$ & 56 & $47.95 \pm 14.66$ \\
9 & $43.57 \pm 10.00$ & 33 & $43.14 \pm 13.86$ & 57 & $48.00 \pm 15.88$ \\
10 & $48.10 \pm 14.57$ & 34 & $56.91 \pm 8.12$ & 58 & $47.87 \pm 19.26$ \\
11 & $50.84 \pm 18.13$ & 35 & $59.89 \pm 12.28$ & 59 & $54.07 \pm 17.13$ \\
12 & $44.73 \pm 9.68$ & 36 & $43.22 \pm 17.04$ & 60 & $38.40 \pm 10.65$ \\
13 & $38.45 \pm 20.39$ & 37 & $59.58 \pm 18.92$ & 61 & $74.13 \pm 17.35$ \\
14 & $50.46 \pm 15.69$ & 38 & $48.71 \pm 12.92$ & 62 & $93.07 \pm 13.35$ \\
15 & $60.66 \pm 15.48$ & 39 & $44.56 \pm 10.93$ & 63 & $71.43 \pm 15.29$ \\
16 & $64.00 \pm 14.15$ & 40 & $41.74 \pm 15.68$ & 64 & $64.52 \pm 15.33$ \\
17 & $40.88 \pm 15.13$ & 41 & $52.31 \pm 13.51$ & 65 & $62.47 \pm 13.72$ \\
18 & $42.07 \pm 4.39$ & 42 & $39.88 \pm 9.35$ & 66 & $59.60 \pm 10.39$ \\
19 & $49.78 \pm 8.05$ & 43 & $39.96 \pm 12.39$ & 67 & $71.15 \pm 13.63$ \\
20 & $46.10 \pm 23.43$ & 44 & $48.89 \pm 14.93$ & 68 & $49.63 \pm 14.02$ \\
21 & $43.24 \pm 10.79$ & 45 & $48.33 \pm 15.44$ & 69 & $41.43 \pm 15.69$ \\
22 & $48.38 \pm 15.08$ & 46 & $47.07 \pm 7.58$ & 70 & $41.81 \pm 15.88$ \\
23 & $48.28 \pm 14.57$ & 47 & $34.38 \pm 16.35$ & 71 & $57.01 \pm 12.08$ \\
24 & $52.43 \pm 14.03$ & 48 & $40.14 \pm 11.95$ & 72 & $45.97 \pm 13.52$ \\
\hline
\end{tabular}


Table 2. The simulation results of the four evaluated control schemes with different amounts of added noise showing the achieved MAE.

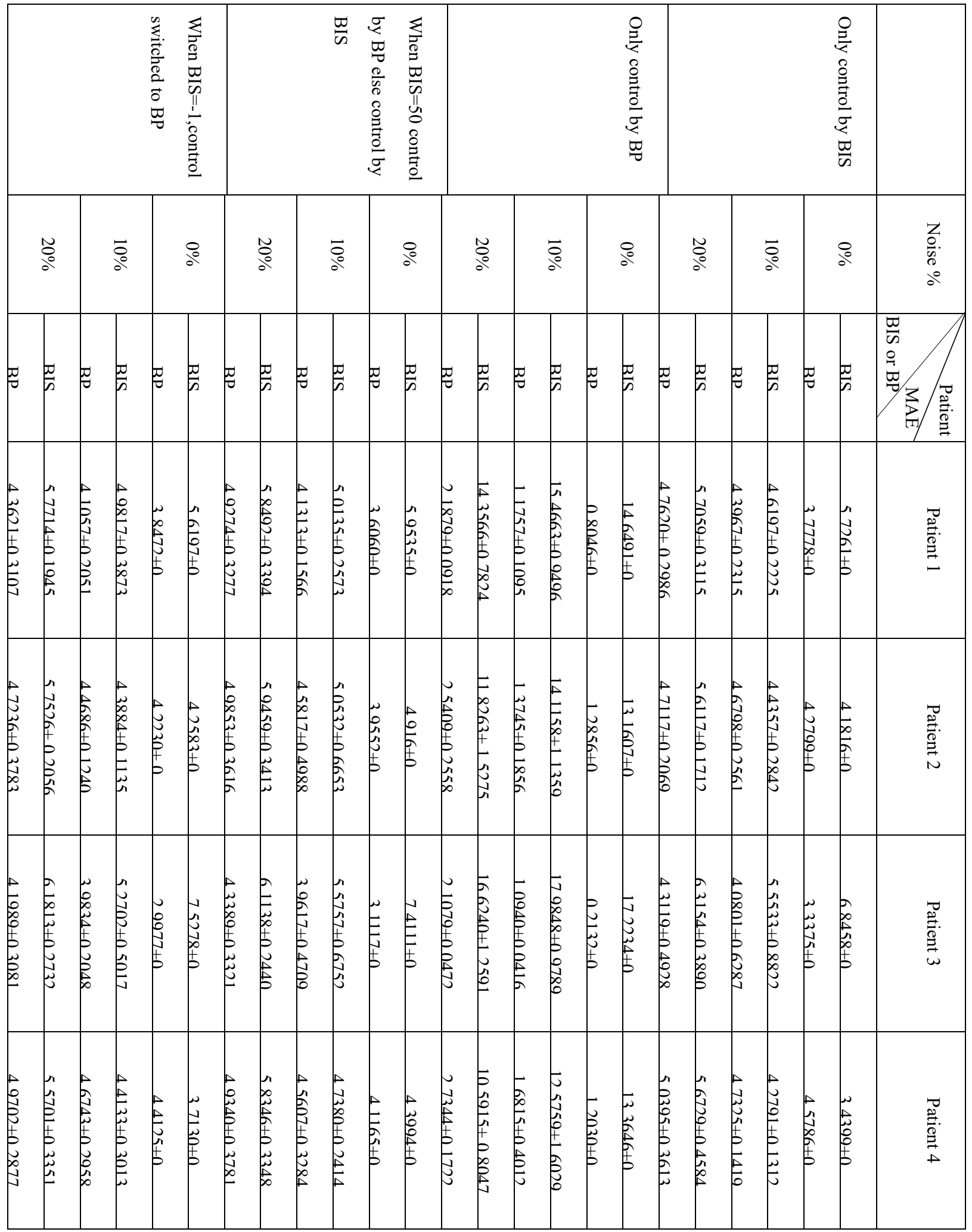




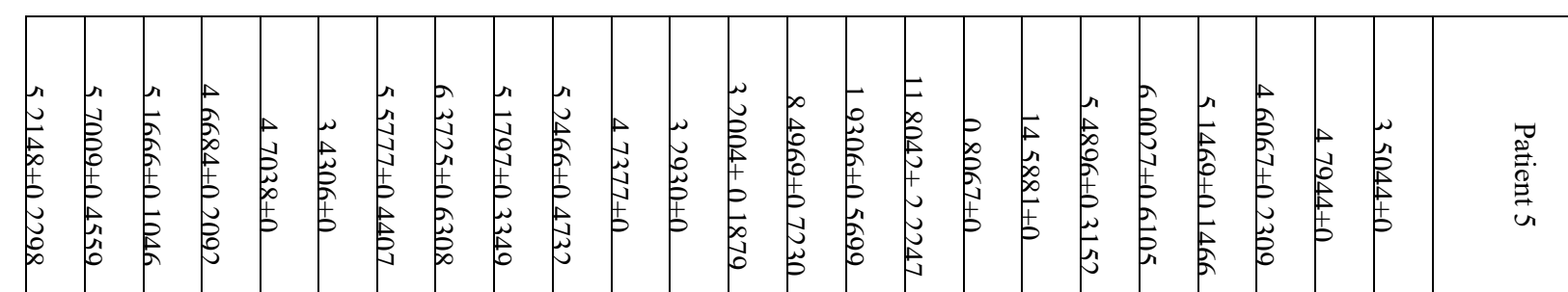

$\mathrm{C}$

$\mathrm{O}$

$\mathrm{Z}$

$\mathrm{OH}$

$\mathrm{Z}$

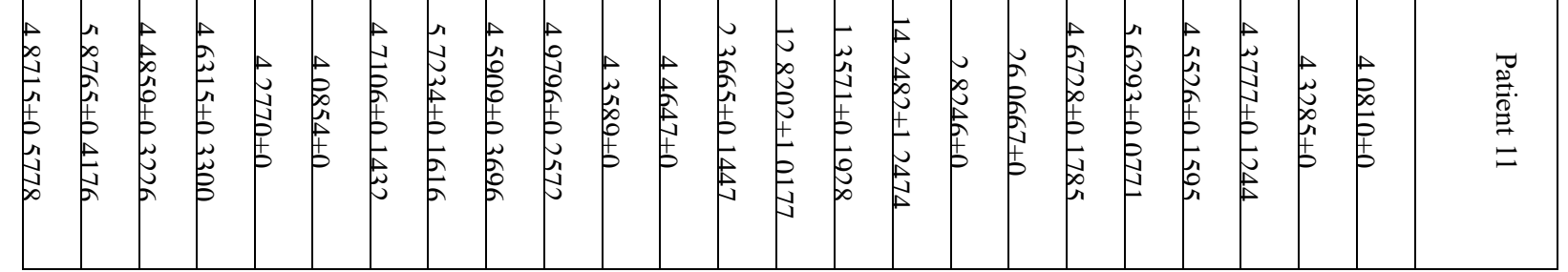




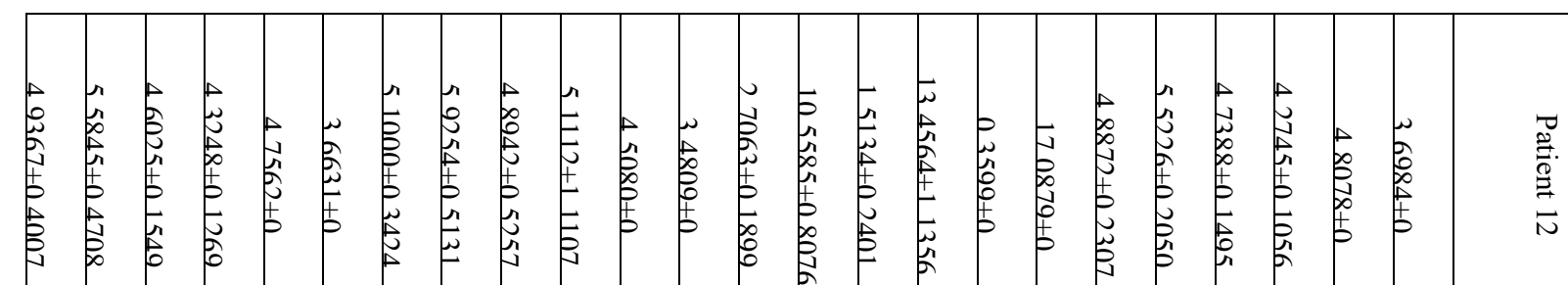

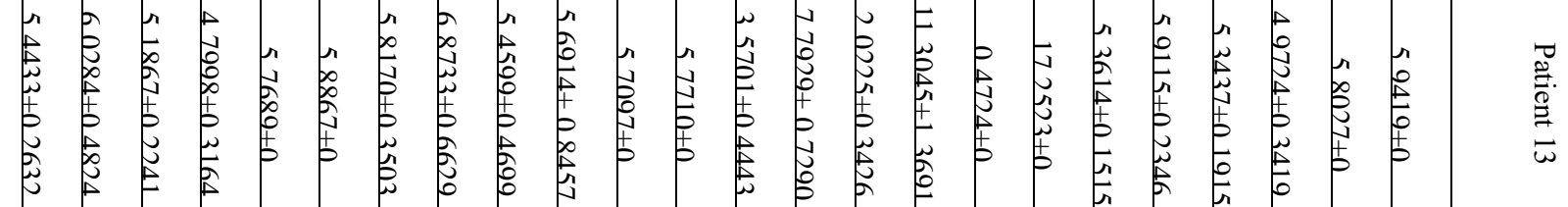
点

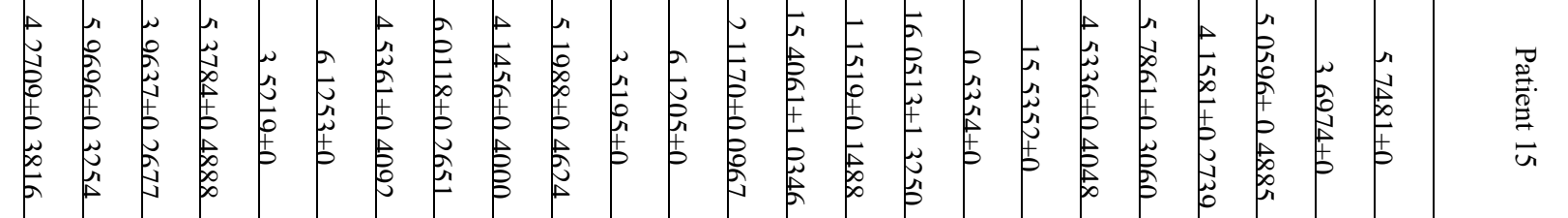

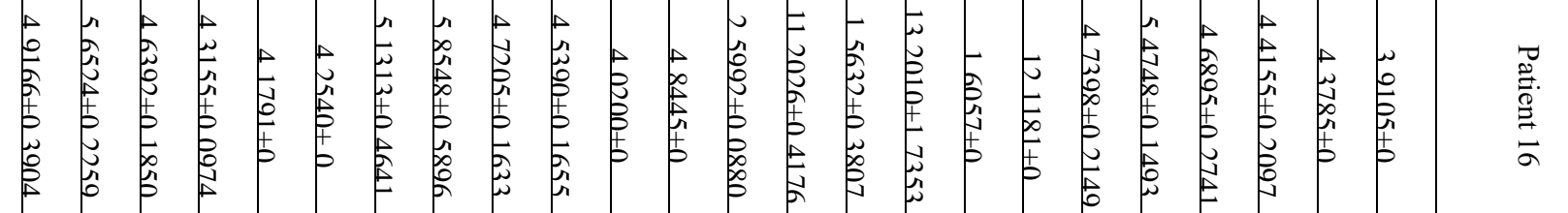

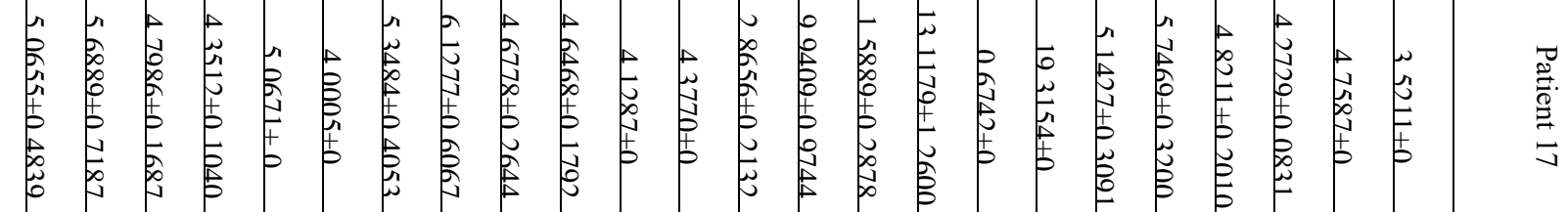

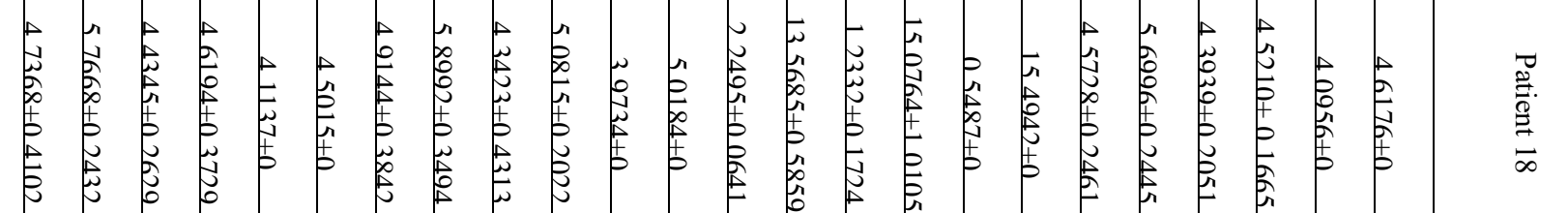




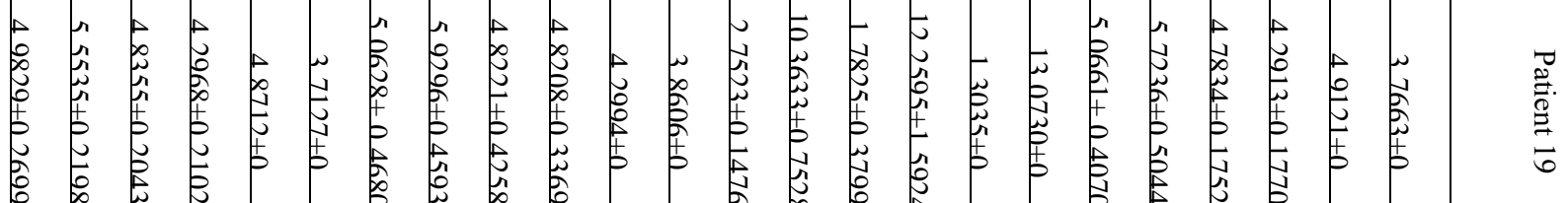

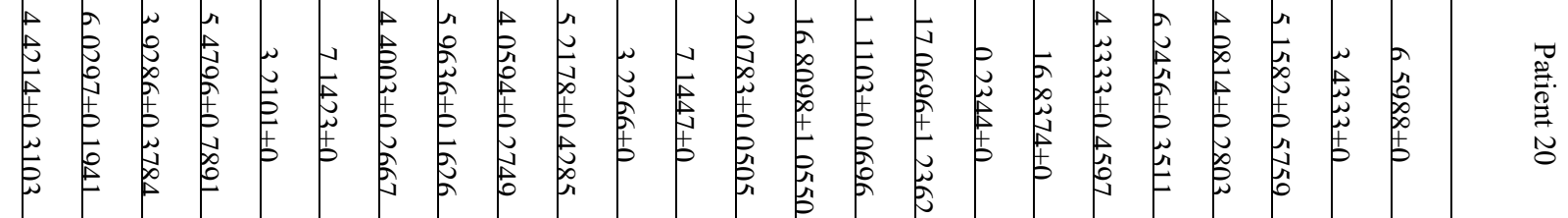

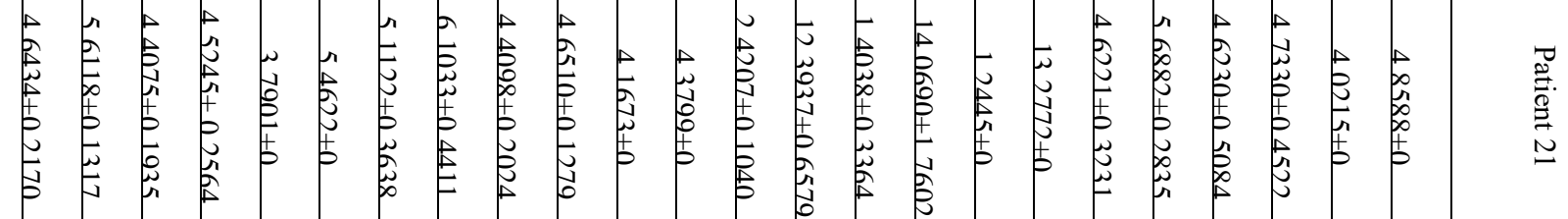

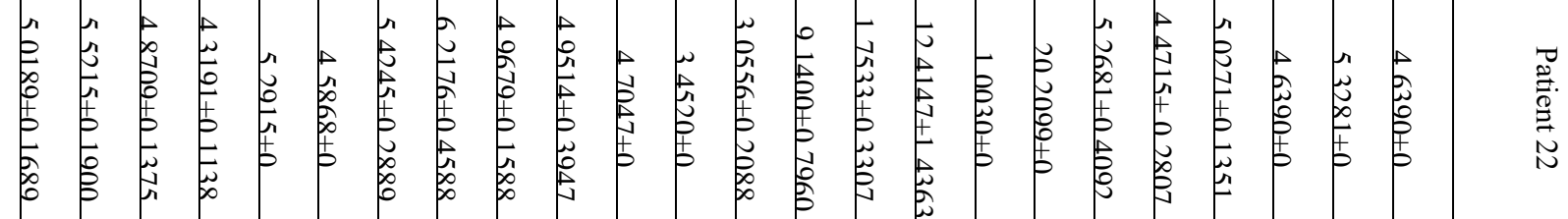

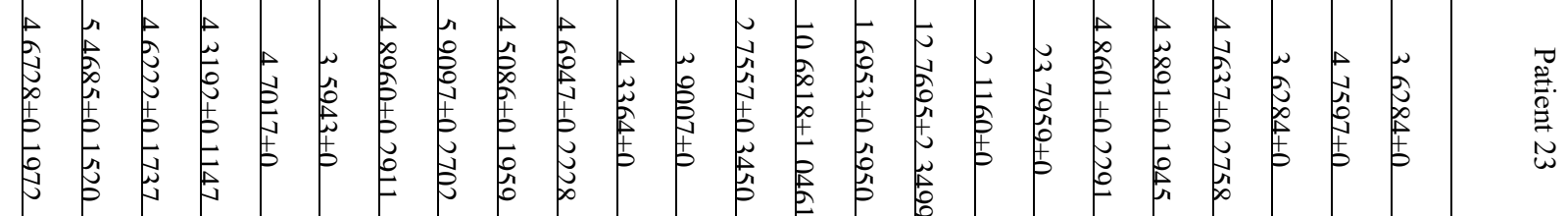

点

$\mathrm{E}$ 


\begin{tabular}{|c|c|c|c|c|c|c|c|c|c|c|c|c|c|c|c|c|c|c|c|c|c|c|c|c|}
\hline 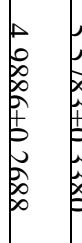 & 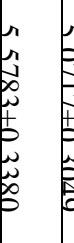 & $\begin{array}{l}n \\
0 \\
0 \\
0 \\
0 \\
0 \\
0 \\
0 \\
0 \\
0\end{array}$ & 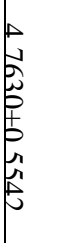 & $\begin{array}{l}n \\
0 \\
0 \\
0 \\
0 \\
0 \\
0\end{array}$ & 官 & $\begin{array}{l}n \\
0 \\
0 \\
0 \\
0 \\
0 \\
5 \\
5 \\
5\end{array}$ & 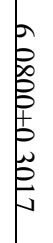 & 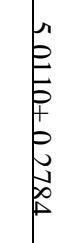 & $\begin{array}{l}0 \\
0 \\
0 \\
0 \\
0 \\
0 \\
0 \\
0 \\
0 \\
0\end{array}$ & 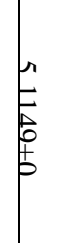 & $\begin{array}{l}0 \\
0 \\
0 \\
0 \\
0 \\
0 \\
0\end{array}$ & 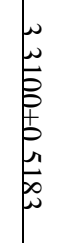 & $\begin{array}{l}\infty \\
0 \\
0 \\
0 \\
E \\
0 \\
0 \\
E \\
E\end{array}$ & 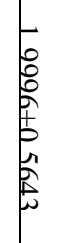 & 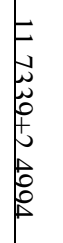 & 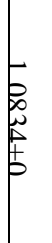 & 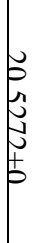 & $\begin{array}{l}0 \\
0 \\
0 \\
0 \\
0 \\
0 \\
0 \\
0 \\
0 \\
0\end{array}$ & $\begin{array}{l}n \\
\vdots \\
\vdots \\
\vdots \\
0 \\
0 \\
\vdots \\
\vdots\end{array}$ & $\begin{array}{l}0 \\
0 \\
0 \\
0 \\
0 \\
0 \\
0 \\
0 \\
0\end{array}$ & 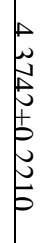 & 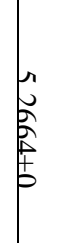 & 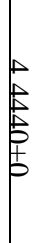 & 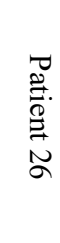 \\
\hline 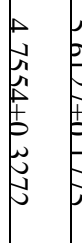 & 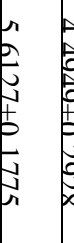 & 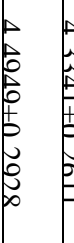 & 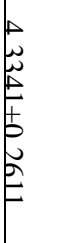 & $\begin{array}{l}0 \\
0 \\
0 \\
0 \\
0 \\
0 \\
0 \\
0\end{array}$ & 舟 & $\begin{array}{l}e_{0} \\
0 \\
0 \\
0 \\
0 \\
0 \\
0 \\
0 \\
0\end{array}$ & 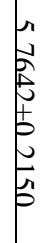 & $\begin{array}{l}b \\
0 \\
0 \\
\hat{1} \\
0 \\
0 \\
0 \\
0 \\
0\end{array}$ & 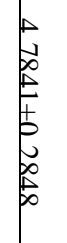 & $\begin{array}{l}b \\
0 \\
0 \\
0 \\
0 \\
0\end{array}$ & $\begin{array}{l}0 \\
0 \\
0 \\
0 \\
0 \\
0\end{array}$ & $\begin{array}{l}\vec{E} \\
\vec{E} \\
E \\
E \\
E\end{array}$ & 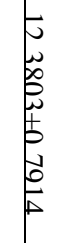 & 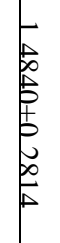 & 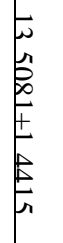 & 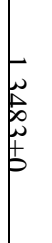 & $\begin{array}{l}\mid \\
b \\
0 \\
0 \\
0 \\
0 \\
0 \\
0\end{array}$ & 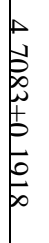 & $\begin{array}{l}n \\
n \\
0 \\
0 \\
0 \\
0 \\
0 \\
0 \\
5\end{array}$ & 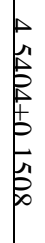 & 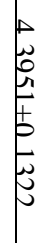 & $\begin{array}{l}B \\
0 \\
0 \\
0 \\
0 \\
0\end{array}$ & 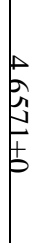 & 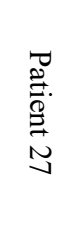 \\
\hline 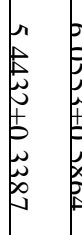 & 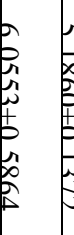 & 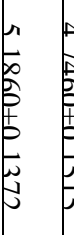 & 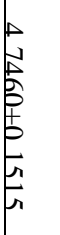 & 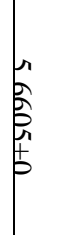 & 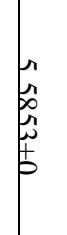 & $\begin{array}{l}n \\
0 \\
0 \\
0 \\
0 \\
0 \\
0 \\
0 \\
0 \\
n\end{array}$ & 等 & $\begin{array}{l}n \\
0 \\
0 \\
0 \\
0 \\
0 \\
0 \\
0 \\
0\end{array}$ & 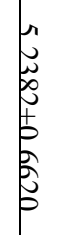 & $\begin{array}{l}n \\
0 \\
0 \\
0 \\
0 \\
0\end{array}$ & $\begin{array}{l}n \\
0 \\
0 \\
0 \\
0 \\
0\end{array}$ & $\begin{array}{l}0 \\
0 \\
0 \\
0 \\
0 \\
0 \\
0 \\
0 \\
0\end{array}$ & $\begin{array}{l}1 \\
0 \\
0 \\
0 \\
0 \\
2 \\
2 \\
2 \\
0\end{array}$ & 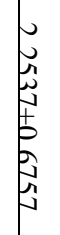 & $\begin{array}{l}\vec{B} \\
\text { J } \\
\mathbf{J} \\
\mathrm{J} \\
\mathrm{t} \\
\mathrm{J} \\
\mathrm{J} \\
\mathrm{J}\end{array}$ & $\begin{array}{l}0 \\
0 \\
0 \\
0 \\
0 \\
0\end{array}$ & 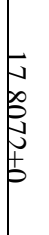 & 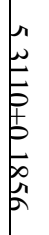 & $\begin{array}{l}n \\
0 \\
0 \\
0 \\
0 \\
0 \\
0 \\
0 \\
0\end{array}$ & $\begin{array}{l}n \\
0 \\
0 \\
0 \\
0 \\
0 \\
0 \\
0 \\
0\end{array}$ & 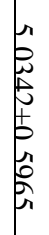 & $\begin{array}{l}n \\
b \\
\vdots \\
\\
\end{array}$ & $\begin{array}{l}n \\
5 \\
\infty \\
0 \\
0 \\
0 \\
0\end{array}$ & 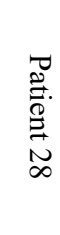 \\
\hline $\begin{array}{l}1 \\
0 \\
0 \\
0 \\
0 \\
0 \\
0 \\
0 \\
\infty \\
0 \\
0\end{array}$ & 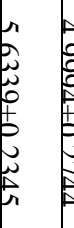 & 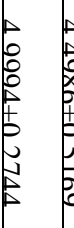 & 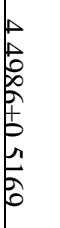 & 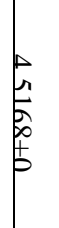 & a & $\begin{array}{l}3 \\
0 \\
0 \\
0 \\
0 \\
0 \\
0 \\
0 \\
\vdots \\
\vdots \\
0\end{array}$ & 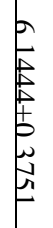 & 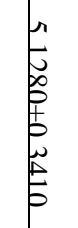 & 至 & 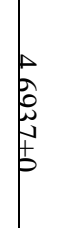 & $\begin{array}{l}0 \\
0 \\
0 \\
0 \\
0 \\
0\end{array}$ & 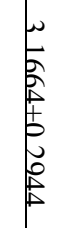 & 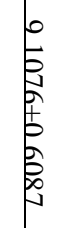 & 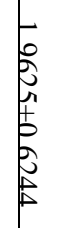 & 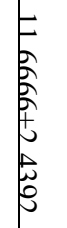 & 焉 & 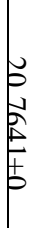 & $\begin{array}{l}n \\
j \\
0 \\
0 \\
0 \\
0 \\
j \\
j\end{array}$ & $\begin{array}{l}0 \\
0 \\
0 \\
0 \\
0 \\
0 \\
\vdots \\
\vdots \\
0 \\
0\end{array}$ & $\begin{array}{l}b_{0} \\
0_{0} \\
0 \\
0 \\
0 \\
0 \\
0 \\
0\end{array}$ & $\begin{array}{l}\text { b } \\
\text { b } \\
b \\
t \\
b \\
b \\
b\end{array}$ & 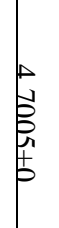 & $\begin{array}{l}\omega \\
\vec{b} \\
0 \\
0 \\
t \\
t\end{array}$ & 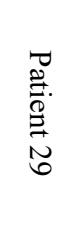 \\
\hline $\begin{array}{l}n \\
5 \\
0 \\
0 \\
0 \\
0 \\
0 \\
0\end{array}$ & 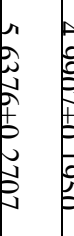 & 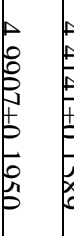 & 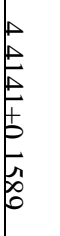 & 离 & 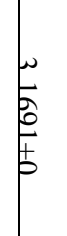 & 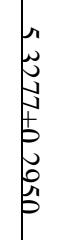 & 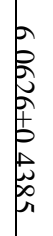 & 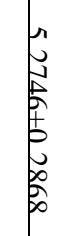 & 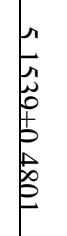 & $\begin{array}{l}1 \\
0 \\
0 \\
0 \\
0 \\
0 \\
0\end{array}$ & $\begin{array}{l}0 \\
0 \\
0 \\
0 \\
0 \\
0 \\
0 \\
0\end{array}$ & 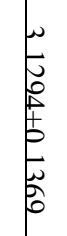 & 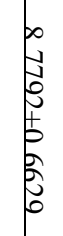 & $\begin{array}{l}- \\
0 \\
0 \\
0 \\
0 \\
0 \\
0 \\
0 \\
0 \\
0 \\
0\end{array}$ & $\begin{array}{l}E \\
E \\
E \\
E \\
E \\
0 \\
0\end{array}$ & 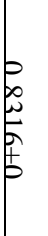 & 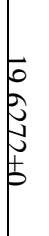 & 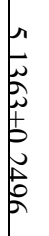 & 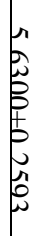 & $\begin{array}{l}n \\
0 \\
0 \\
0 \\
0 \\
0 \\
0 \\
0 \\
0 \\
0\end{array}$ & 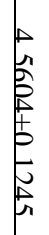 & $\begin{array}{l}0 \\
0 \\
0 \\
0 \\
0 \\
0\end{array}$ & & 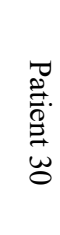 \\
\hline
\end{tabular}


Table 3. The results of mean $\mathrm{MAE} \pm \mathrm{SD}$ values of each control scheme of 30 patients in comparison with each control scheme with the third control scheme

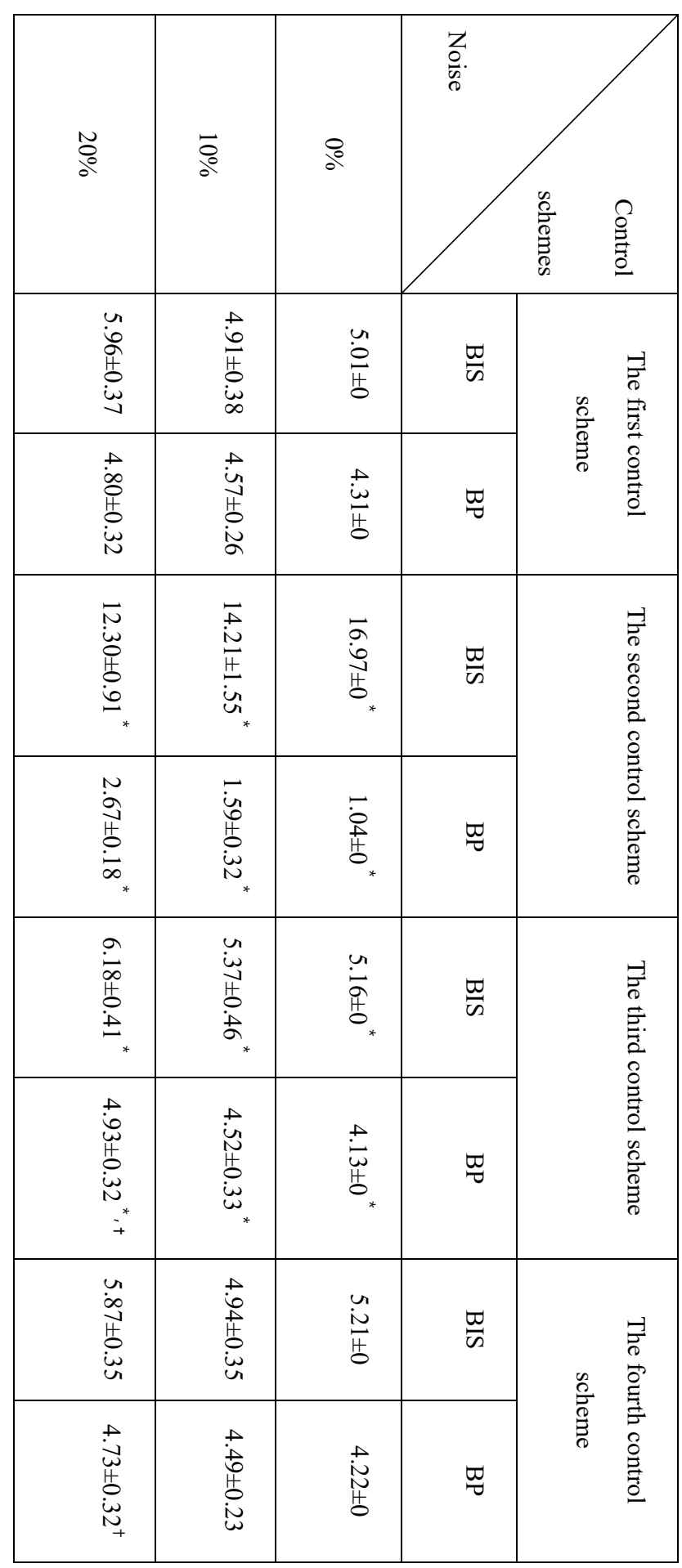

* Represent the second and the third control schemes have significant differences.

† Represent the third and the fourth control schemes have significant differences. 